Supporting Information

\title{
Complex Resonant Scattering Behavior in the Surface Plasmon Resonance Imaging Microscopy of Single Gold Nanorods
}

Yunshan Fan, Yara Aceta, ${ }^{\dagger}$ Esther Hessong, Athena Bengston, Laris A. Biageyian, Quan P.

Huynh and Robert M. Corn*

Department of Chemistry, University of California-Irvine, Irvine, California 92697 USA

\section{Corresponding Author}

*Email: rcorn@uci.edu

Present Address

${ }^{\dagger}$ IETR UMR CNRS 6164 Site Université de Nantes, F-44322 Nantes Cedex 3, France 


\section{Experimental Method}

Chemicals and Materials. All the following chemicals and materials were used as received from the manufacturers. Potassium phosphate monobasic $\left(\mathrm{KH}_{2} \mathrm{PO}_{4}\right.$, Fisher Chemical, 99.4\%), dipotassium phosphate $\left(\mathrm{K}_{2} \mathrm{HPO}_{4}\right.$, Fisher Chemical, 99.4\%), 11-amino-1-undecanethiol hydrochloride (MUAM, Dojindo Molecular Technologies, Inc., Gaithersburg, MD, USA, $\geq 90.0 \%$ ), gold nanorods (AuNRs) with peak absorbance at 670/816/980 nm (sodium citrate capped, dispersed in Milli-Q water, NanoComposix, Inc. San Diego, CA). All aqueous solutions were prepared using deionized water $(>18 \mathrm{M} \Omega \cdot \mathrm{cm})$ from a Millipore Milli-Q water purification system.

Substrate Preparation. Substrates for all SPRI experiments were No. 1.5 coverslips (Fisherbrand, Pittsburgh, PA, USA) coated with a $1 \mathrm{~nm}$ adhesion layer of chromium, followed by $45 \mathrm{~nm}$ of gold using a thermal evaporator. Positively charged alkanethiol (11-amino-1-undecanethiol, MUAM) monolayers were prepared on the gold-covered coverslips by immersing the gold substrates in a $1 \mathrm{mM}$ ethanolic MUAM solution for $12 \mathrm{~h}$.

Optical Setup and Image Acquisition. The detailed description of the construction of the singlenanoparticle SPRI microscope is described in a previous report. ${ }^{1}$ Briefly, the microscope was built into the frame of an IX51 inverted microscope (Olympus, Tokyo, Japan). A $1 \mathrm{~mW} 814 \mathrm{~nm}$ diode laser (Melles Griot, Carlsbad, CA) was expanded and collimated using a spatial filter (Newport Corp., Newport Beach, CA). The beam was then polarized and focused with a lens $(f=200 \mathrm{~mm})$ onto the back focal plane of a $100 \times$ 1.49 NA oil immersion microscope objective (Olympus). The focused beam was directed upward near the edge of the objective by a gold-coated knife-edge mirror (Thorlabs, Newton, NJ, USA) that was mounted on an $\mathrm{X}-\mathrm{Y}$ micrometer to adjust the incident angle on the sample. The reflected image was then acquired by an Andor Neo sCMOS camera (South Windsor, CT, USA) by accumulating 30 11-bit, 0.1s exposures. All $\mathrm{Au}$ surfaces were partitioned using adhesive silicone isolation wells (Electron Microscopy Sciences, Hatfield, PA, USA). A10 $\mu \mathrm{L}$ volume of nanorod solution $\left(\sim 10^{9}\right.$ particles $\left./ \mathrm{mL}\right)$ was injected to a well, and the electrostatic adsorption of particles was observed.

UV-Vis Spectroscopy. The UV-visible absorption spectra of the three AuNR samples were acquired on a Lambda 950 spectrophotometer from Perkin Elmer. 


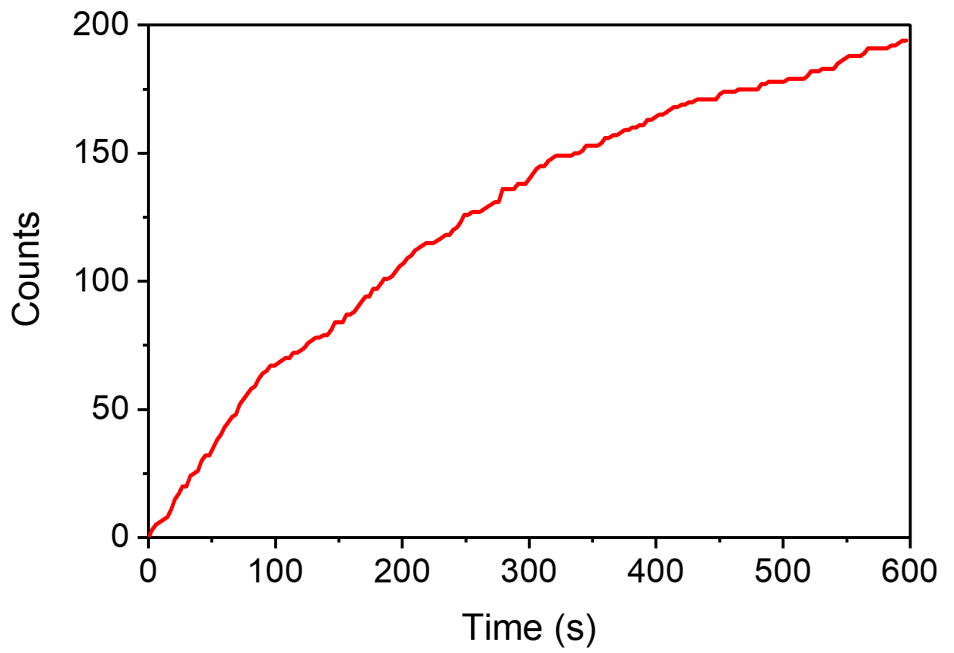

Figure S1. A representative $670 \mathrm{~nm}$ AuNR adsorption curve as a function of time over the course of an SPRI microscopy experiment. 
a)

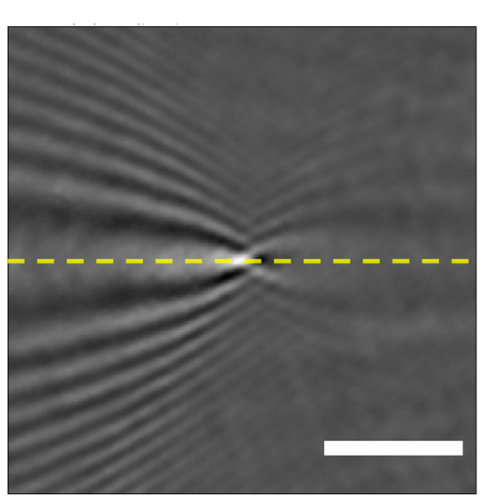

b)

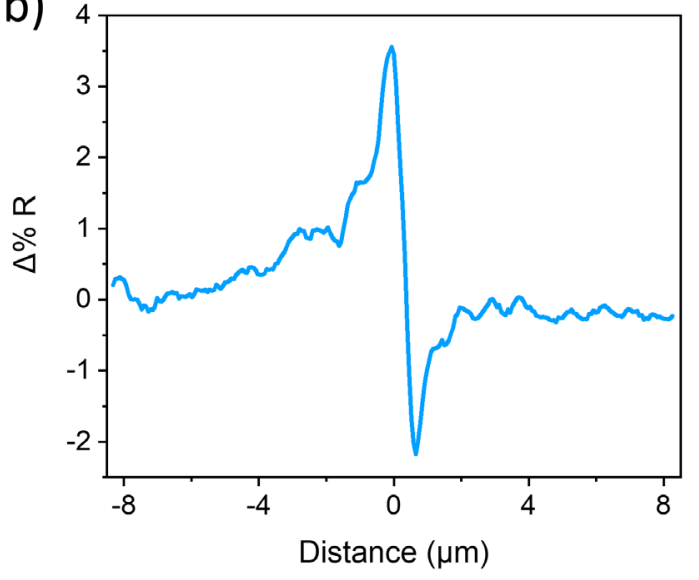

Figure S2. (a) An expanded area view of a $100 \mathrm{~nm}$-diameter spherical Au nanoparticle diffraction pattern. The scale bar represents $5 \mu \mathrm{m}$. (b) Horizontal intensity profile taken through the center of the diffraction pattern (indicated by the yellow horizontal dashed line) in (a). 
Table S1. Parameters used for theoretical calculations of AuNR SPRI diffraction images and center plots with different $M_{R}$ values.

\begin{tabular}{ccccccccccc}
$M_{R}$ & $\sigma^{*}$ & $k_{\text {in }}$ & $k_{\text {sp1 }}$ & $k_{\mathrm{sp} 2}$ & $k_{\mathrm{d} 1}$ & $k_{\mathrm{d} 2}$ & $\left|\alpha_{1}\right|$ & $\left|\alpha_{2}\right|$ & $\phi_{1}$ & $\phi_{2}$ \\
-1 & 0.06 & 10.55 & 10.52 & 10.81 & 0.18 & 0.60 & 0.020 & 0.020 & $1.42 \pi$ & $1.15 \pi$ \\
+0.3 & 0.04 & 10.55 & 10.52 & 10.81 & 0.30 & 0.60 & 0.025 & 0.033 & 0 & $4 \pi / 3$ \\
+1 & 0.16 & 10.55 & 10.52 & 10.81 & 0.30 & 0.90 & 0.035 & 0.035 & $\pi / 3$ & $5 \pi / 3$ \\
\hline
\end{tabular}

* $\sigma$ is the standard deviation in the two-dimensional gaussian point spread function: $O=e^{-\left(x^{2}+y^{2}\right) /\left(2 \sigma^{2}\right)}$.

\section{References}

(1) Halpern, A. R.; Wood, J. B.; Wang, Y.; Corn, R. M. Single-Nanoparticle Near-Infrared Surface Plasmon Resonance Microscopy for Real-Time Measurements of DNA Hybridization Adsorption. ACS Nano 2014, $8,1022-1030$. 